\title{
Estratégia reprodutiva de Cochlospermum orinocense (Kunth) Steud.: fenologia, biologia floral e sistema de cruzamento em uma espécie pioneira de florestas na Amazônia
}

\author{
PAULO SÉRGIO DE FIGUEIREDO ${ }^{1,3}$, ÉVILLE KARINA MACIEL DELGADO RIBEIRO², \\ DINNIE MICHELLE ASSUNÇÃO LACERDA ${ }^{1}$ e EMÍLIA CRISTINA GIRNOS ${ }^{1}$
}

(recebido: 18 de fevereiro de 2009; aceito: 12 de setembro de 2009)

\begin{abstract}
Reproductive strategy of Cochlospermum orinocense (Kunth) Steud.: phenology, floral biology and breeding system of an Amazonian forest pioneer species). Cochlospermum orinocense (Kunth) Steud. is an arboreal species found in forest edges, canopy gaps and secondary forests. Phenology, floral biology and breeding system data was gathered at the edge of an 80 ha primary terra-firme Amazonian forest fragment in the São Luís Island, Maranhão state. Cochlospermum orinocense is a deciduous species which flower annually, showing intense and synchronized flowering early in the dry season. Its hermaphrodite and slightly zygomorphic flowers are arranged in terminal inflorescences and last only one day. Each ovary has on average 250 ovules and the androecium about 160 poricidal dehiscence anthers presenting 81.6\% viable pollen and $\mathrm{P} / \mathrm{O}$ ratios of 16,019.2. Anthesis is diurnal and the flowers are pollinated mainly by solitary bees of the species Centris spp. Cochlospermum orinocense is self-compatible (ISI $=0.98)$ with high reproductive efficacy $(\mathrm{RE}=0.81)$ and reduced or null apomixis. Clustered distribution in deciduous trees together with intense and synchronized flowering in the dry season leads to highly efficient pollination. Fecundity is thus limited to resources constraints and not by pollen availability. Geitonogamy, the commonest form of endogamy, may assure the reproductive success of isolated individuals in the forest but leads to endogamic depression. The high density clustering often found in this species encourages allogamy and may allow high reproductive efficiency and higher fecundity as endogamic depression is minimized.
\end{abstract}

Key words - Cochlospermum orinocense, geitonogamy, inbreeding depression, mixed mating system

RESUMO - (Estratégia reprodutiva de Cochlospermum orinocense (Kunth) Steud.: fenologia, biologia floral e sistema de cruzamento em uma espécie pioneira de florestas na Amazônia). Cochlospermum orinocense (Kunth) Steud. é uma espécie arbórea freqüente em clareiras, na borda da floresta e em florestas secundárias na Amazônia. Dados da fenologia, biologia floral e sistema de cruzamento foram obtidos em indivíduos na borda de um fragmento de 80 ha de floresta Amazônica primária, de terra firme, na ilha de São Luís, MA. Cochlospermum orinocense é uma espécie decídua com floração anual precoce, intensa e sincronizada na estação seca. Suas flores hermafroditas e levemente zigomorfas estão dispostas em inflorescências terminais e têm duração de um dia. Cada ovário possui em média 250 óvulos e o androceu é composto por cerca de 160 anteras, de deiscência poricida, apresentando 81,6\% de pólen viável e razão P/O de 16.019,2. A antese é diurna e as flores são polinizadas predominantemente por abelhas solitárias Centris spp. A espécie é autocompatível (IAI = 0,98) com alta eficácia reprodutiva $(E R=0,81)$, sendo a apomixia reduzida ou nula. Distribuição agrupada e floração intensa e sincronizada na seca, em árvores decíduas, induzem alta eficiência na polinização, sendo a fecundidade limitada pelos recursos da planta e não por pólen. Geitonogamia, a forma mais freqüente de endogamia, pode assegurar o sucesso reprodutivo em indivíduos isolados na floresta, mas induz forte depressão endogâmica. Alta densidade e a forma agrupada com que a espécie predominantemente ocorre, favorecendo a alogamia, podem permitir alta eficiência reprodutiva e fecundidade ao minimizar a depressão endogâmica.

Palavras-chave - Cochlospermum orinocense, depressão endogâmica, geitonogamia, sistema de cruzamento misto

\section{Introdução}

Cochlospermum é um pequeno gênero pantropical com cerca de 15 espécies, a maioria das quais ocorre

\footnotetext{
1. Universidade Federal do Maranhão, Departamento de Biologia, Avenida dos Portugueses, s/nº, Campus Universitário do Bacanga, 65085-580 São Luís, MA, Brasil.

2. Universidade Federal de Pernambuco, Departamento de Botânica, Rua Prof. Moraes Rego, s/no, Cidade Universitária, 50372-970 Recife, PE, Brasil.

3._Autor para correspondência: paulosfigueiredo@uol.com.br
}

na América do Sul. Em geral são árvores de pequeno a médio porte, decíduas, que florescem quando sem folhas produzindo uma grande quantidade de flores grandes, predominantemente amarelo-alaranjadas, hermafroditas, dispostas em inflorescências terminais ou axilares (Woodson et al. 1967).

Cochlospermum orinocense (Kunth) Steud. é uma espécie arbórea pioneira, com 7 a 12 m de altura, que se distribui predominantemente na Amazônia ocorrendo no Brasil, Peru, Bolívia, Equador, Venezuela e Colômbia (USDA 2006). Espécie heliófita, de rápido 
crescimento, exibe alta fecundidade investindo na produção de numerosas sementes pequenas e aladas. Estas características favorecem competição eficiente em ambientes instáveis como clareiras e bordas das florestas de terra firme, seu habitat preferencial. Além de atuar na regeneração natural e na expansão destas florestas, constitui-se também em elemento importante na sucessão secundária em áreas antropicamente desmatadas.

Suas flores, dispostas em inflorescências terminais do tipo panícula, não produzem néctar, são conspícuas, amarelas, pentâmeras e actinomorfas. O gineceu é sincárpico com ovário súpero, globoso, tricarpelar, com muitos óvulos anátropos num único loco, sendo o estilete linear, alongado e recurvado no ápice. No androceu, os numerosos estames são todos férteis e livres entre si, com anteras basifixas e polens tetraloculados dispersos através do poro apical introrso. Os frutos são cápsulas lenhosas deiscentes, trivalvares, com muitas sementes aladas, reniformes, oleaginosas e endospérmicas. O embrião é curvado e os cotilédones grandes e foliáceos (Eichler 1871, Woodson et al. 1967, Keating 1972, Macfarlane 2007).

A presença de anteras poricidas, cujos grãos de pólen são liberados por vibração, indica os principais polinizadores no gênero: abelhas de médio ou grande porte como Centris spp. em C. vitifolium Spreng. (Chapotin et al. 2003) e Centris sp. e Xylocopa sp. em C. regium Pilg. (Noronha \& Gottsberger 1980). Membros do gênero Centris (Apidae) são especialmente importantes como agentes polinizadores de espécies alógamas (Bawa 1974, Frankie et al. 1976, Frankie \& Coville 1979), sistema de cruzamento predominante nas árvores das florestas tropicais (Gentry 1974, Primack 1985, Bawa 1992). Estas abelhas nidificam em troncos caídos, ou no próprio solo, com a maioria das espécies preferindo sítios sombreados na floresta (Frankie et al. 1988) e, dada sua abundância e eficiência como forrageadores entre plantas e distância de vôo de vários quilômetros (Frankie et al. 1976, Frankie et al. 1988), maximizam a eficiência reprodutiva das plantas e o fluxo gênico na população.

Tem sido demonstrado que flores hermafroditas e autoincompatibilidade predominam nas espécies arbóreas alógamas das florestas tropicais (Bawa 1974, 1992, Zapata \& Arroyo 1978, Opler et al. 1980, James et al. 1998, Chaszdon et al. 2003, Knight et al. 2005), sendo a limitação de pólen e/ou a deposição de pólen incompatível as possíveis causas da menor eficiência reprodutiva destas espécies quando comparadas às autocompatíveis (Opler et al. 1980, Knigth et al. 2005). Alógamas autocompatíveis são mais freqüentes nos estágios serais iniciais (Opler et al. 1980) e considera-se que estejam, junto com as espécies dióicas, entre as mais eficientes reprodutivamente nas florestas (Sutherland \& Delph 1984), embora Zapata e Arroyo (1978) não tenham obtido diferenças significativas entre a eficácia reprodutiva de alógamas compatíveis e incompatíveis em floresta secundária na Venezuela.

Alógamas autocompatíveis costumam exibir grau variável de autocompatibilidade e de adaptação à endogamia (Zapata \& Arroyo 1978, James et al. 1998) que podem minimizar o efeito da limitação de pólen. No entanto, sua eficiência reprodutiva pode ser comprometida na dependência da intensidade da depressão endogâmica: a expressão de alelos recessivos letais ou semi-letais na endogamia (Koelewijn 1998, Knigth et al. 2005).

Como a eficiência reprodutiva das plantas tem reflexos diretos na fecundidade, sua magnitude e variação podem ter grandes conseqüências demográficas. Estas conseqüências são potencialmente mais drásticas nas espécies pioneiras que se estabelecem por sementes em resposta a distúrbios no ambiente (Bond 1994, Ashman et al. 2004).

Neste trabalho são descritos alguns aspectos da biologia floral e reprodutiva de $C$. orinocense, incluindo o seu sistema de cruzamento, acrescentando informações sobre a estratégia reprodutiva de espécies pioneiras e seus reflexos na eficiência reprodutiva e fecundidade.

\section{Material e métodos}

O estudo foi realizado em uma reserva particular de 400 ha pertencente à Merck S.A., situada no Município de São José de Ribamar (MA), a leste da ilha de São Luís

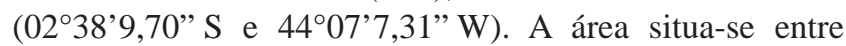
9 e $15 \mathrm{~m}$ de altitude e encontra-se na transição da floresta amazônica para o mangue e a restinga, sendo constituída de um fragmento com cerca de 80 ha de floresta primária de terra firme, sempre-verde, com trechos sazonalmente inundáveis e 120 ha de mangue. O restante é ocupado por florestas secundárias, com cerca de 150 ha, e vegetação de restinga, com aproximadamente 50 ha.

Situadas na transição para o semi-árido, sob clima Aw ou tropical quente e úmido de acordo com a classificação de Köppen, as formações florestais da ilha caracterizam-se como florestas tropicais sazonalmente secas (sensu Pennington et al. 2000), estando submetidas a uma precipitação anual entre 1.600 e 2.400 mm, com excedente hídrico acima de $1.000 \mathrm{~mm}$ entre janeiro e junho, seguida de um período de estiagem de julho a dezembro com deficiência hídrica moderada nos solos (figura 1). Para a elaboração do balanço hídrico consideraram-se os dados históricos dos últimos 10 anos em São Luís, MA. Tanto estes quanto os dados climáticos 


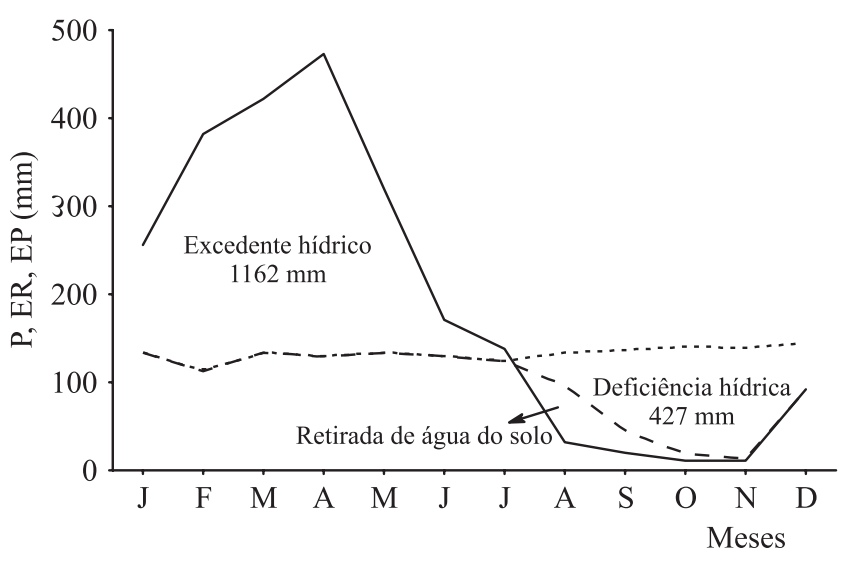

Figura 1. Balanço hídrico do solo segundo ThorntwaiteMather, considerando dados históricos em São Luís, MA. (- = Precipitação (P); - - = Evapotranspiração real (ER); --- = Evapotranspiração potencial (EP)).

Figure 1. Thorntwaite-Mather soil water balance considering historical data in São Luís, MA. (- = Precipitation (P); $--=$ Actual evapotranspiration (ER); $---=$ Potential Evapotranspiration (EP)).

mais detalhados do período de estudo (ano de 2007) foram extraídos dos boletins meteorológicos do Laboratório de Meteorologia da Universidade Estadual do Maranhão.

Dados fenométricos foram obtidos em amostra de 16 indivíduos arbitrariamente selecionados em uma área de $200 \mathrm{~m} \times 80 \mathrm{~m}$ da borda da mata primária de terra firme, utilizando como critério de inclusão a possibilidade de visualização de toda a copa. Os indivíduos foram marcados e numerados sequencialmente sendo observados a cada 15 dias, de abril de 2007 a janeiro de 2008. O desenvolvimento vegetativo (dinâmica da produção e abscisão de folhas) foi estimado com base no método de Fournier (1974) modificado, utilizando-se como índice a média da proporção da copa com folhas, mas adotando-se 0,1 como intervalo de classe. $\mathrm{O}$ desenvolvimento reprodutivo foi estimado contando-se o número de inflorescências e de inflorescências com frutos por indivíduo. Os dados fenométricos da proporção da copa com folhas, número de inflorescências e de inflorescência com frutos por planta foram correlacionados com a precipitação mensal por regressão linear simples, estimando-se o coeficiente de correlação de Pearson (Zar 1996).

Dada a maior facilidade de acesso à copa das árvores, os dados sobre a biologia floral e sistema de cruzamento foram obtidos em amostra de oito indivíduos ocorrendo naturalmente em áreas desmatadas, mas com floresta secundária em regeneração, a cerca de $500 \mathrm{~m}$ da borda da floresta primária. O número total de flores (botões e flores abertas) e de frutos por inflorescência, assim como o número de flores abertas por dia, por inflorescência, foram estimados no auge da floração e frutificação, respectivamente, em amostra de quatro inflorescências por indivíduo, em cinco indivíduos. Dados morfológicos e morfométricos nas flores foram obtidos em amostra de 10 botões e flores selecionadas em três indivíduos, as quais foram fixadas em etanol $70 \%$. As peças florais foram medidas com auxílio de paquímetro digital e os dados morfométricos obtidos sob estereomicroscópio.

A longevidade das flores foi estimada em amostras de 54 flores expostas e 40 flores ensacadas, com sacos de organza transparente, em três indivíduos. Elas foram observadas, a partir da pré-antese, a cada duas horas durante 24 horas. A longevidade de flores fecundadas foi determinada pela duração de flores, expostas ao polinizador, que efetivamente originaram frutos. Determinou-se a longevidade de flores não fecundadas pela duração das flores expostas que abortaram precocemente, dado que as ensacadas manifestaram também senescência e aborto precoce. A longevidade foi expressa graficamente como porcentagem de flores abertas, computando-se a porcentagem de aborto/hora do total de flores que abortaram.

A viabilidade polínica foi estimada por meio da coloração do citoplasma pelo carmim acético (Radford et al. 1974) e a receptividade do estigma foi avaliada utilizando-se permanganato de potássio 0,25\% (Robinsonh 1924). Os testes de receptividade foram realizados em amostras de 10 flores em três indivíduos, coletadas a cada intervalo de duas horas a partir da antese, das 5 h00 até às 15 h00. O número de grãos de pólen/flor foi obtido com auxílio de câmara de Neubauer (Lloyd 1972, Maêda 1985) em amostras de cinco flores/indivíduo, em três indivíduos, sendo estimada a razão P/O segundo a metodologia de Cruden (1977).

O sistema de cruzamento da espécie foi analisado através de tratamentos de polinização aberta (polinização natural), autopolinização espontânea, autopolinização manual, xenogamia, geitonogamia e apomixia (Radford et al. 1974). Nos tratamentos, os botões em fase de pré-antese foram isolados e a inflorescência coberta com sacos de organza branco transparente, exceto na polinização natural, removendo-se o restante dos botões da inflorescência. O tipo de polinização desejada foi realizada logo após a antese, entre 8h00 e 10h00, sendo as flores emasculadas e a inflorescência novamente ensacada. Utilizou-se uma amostra de 25 flores, por tratamento, selecionadas em cinco indivíduos. Foram calculados a eficácia reprodutiva (ER) e o índice de autoincompatibilidade (IAI) de acordo com Zapata \& Arroyo (1978).

Nos frutos formados em cada tratamento determinou-se o número e a massa das sementes maduras, sendo as sementes pesadas individualmente em balança de precisão. A proporção de frutos formados em cada tratamento (sucesso reprodutivo) foi comparada pelo teste do Qui-quadrado e o número e massa das sementes pelo teste de Kruskall-Wallis seguido do U Mann-Whitney, ambos com $P \leq 5 \%$ (Zar 1996).

Os dados de freqüência de visitas por hora, assim como a duração, comportamento de visita das abelhas e local de contato com pólen e estigma, foram obtidos através de observações diretas nas flores de três indivíduos durante o pico de floração, totalizando 12 horas de observação contínua. Espécimes das abelhas foram capturados com auxílio de rede 
entomológica e rede de neblina (utilizadas para a captura de aves), sendo posteriormente montados, etiquetados, identificados e depositados na coleção do Laboratório de Estudos sobre Abelhas (LEA), da Universidade Federal do Maranhão.

\section{Resultados}

O ano de 2007 teve um volume de chuvas de $1.160 \mathrm{~mm}$, sendo bem inferior à média histórica de $1.520 \mathrm{~mm}$ de precipitação anual. Além da precipitação sazonal, sazonalidade climática é imposta também pela variação na radiação solar que aumenta significativamente na seca, provocando um aumento nas temperaturas médias e redução na umidade relativa fatos que, associados ao aumento do número de dias sem chuvas, tornam a primavera a estação mais quente e seca do ano (figuras 1 e 2).

Em Cochlospermum orinocense, a proporção de folhas na copa correlaciona-se positivamente com a precipitação do período $(r=0,76, P=0,01)$. A espécie é decídua e a abscisão anual das folhas no inverno, no final do período chuvoso, marca o início da dormência das gemas vegetativas cuja quebra, evidenciada pela rebrota vigorosa, ocorre no início das chuvas de verão. A queda das folhas e a dormência precedem a floração anual, a qual se correlaciona com o decréscimo da precipitação da transição inverno/primavera $(r=-0,79$, $P=0,21$ ), precocemente na seca (figura 3 ). O padrão de floração é cornucópia (Gentry 1974): floração intensa e sincronizada, cada indivíduo produzindo cerca de 2.500 flores (tabela 1) durante um período de 8 a 12 semanas (figura 3). O “display floral” no auge da floração é de aproximadamente 73 flores abertas/indivíduo/dia (tabela 1).

A frutificação ocorre na primavera, no auge da seca (figura 3), sendo negativamente correlacionada com a precipitação $(r=-0,85, P=0,001)$. Cada indivíduo produz aproximadamente 240 frutos (tabela 1), sendo a razão fruto/flor igual a 0,094 . Os frutos produzem em média 57,91 \pm 22,8 sementes sob polinização natural (tabela 1), sendo a razão semente/óvulo igual a 0,23 . Suas muitas sementes aladas, cerca de 13.800 por indivíduo (tabela 1), são dispersas ao final da seca, na primavera, e durante as primeiras chuvas do verão (figura 3).

As flores de C. orinocense estão dispostas em inflorescências compostas, terminais, indeterminadas e

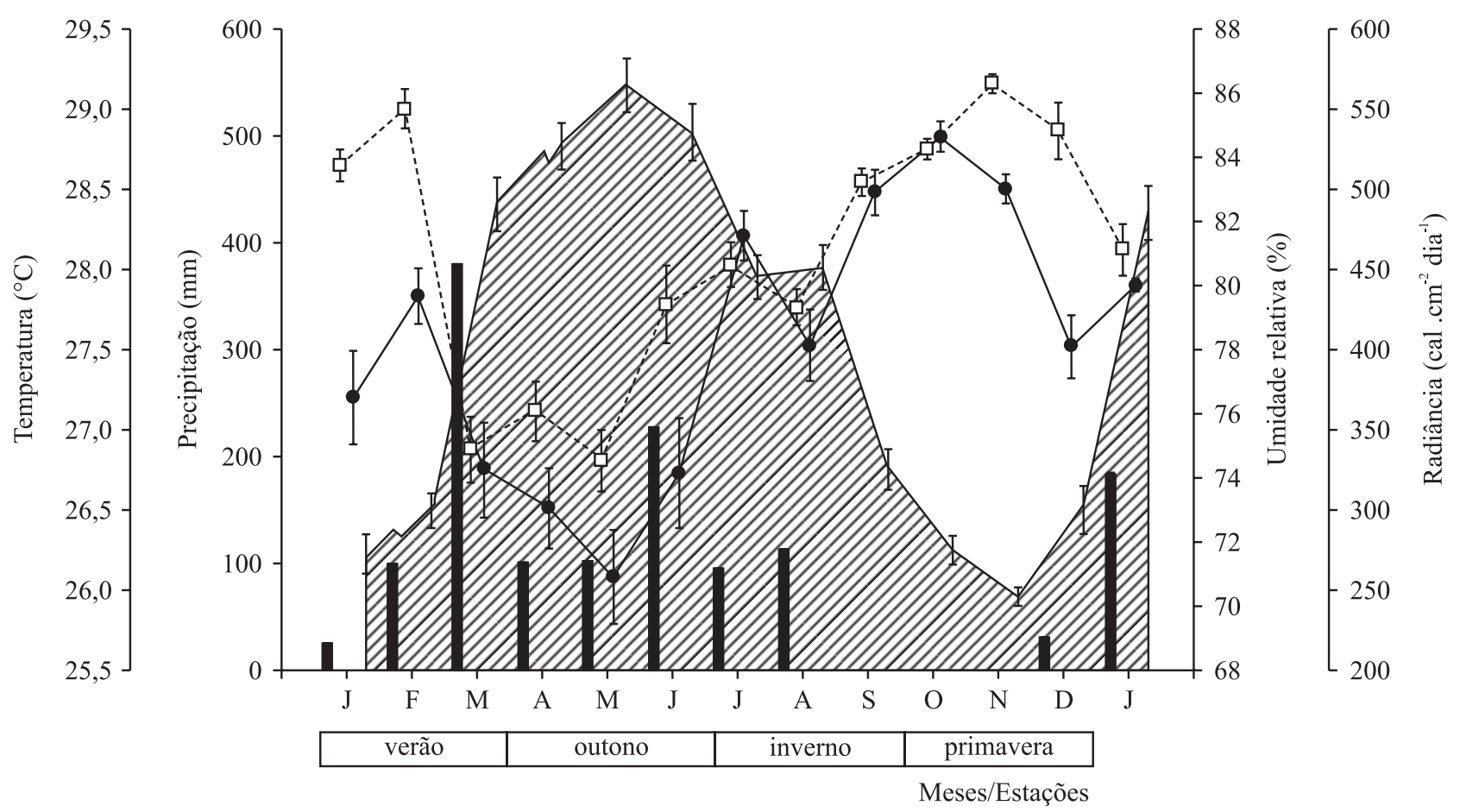

Figura 2. Dados climáticos em São Luís, MA, de janeiro de 2007 a janeiro de 2008. ( $=$ precipitação; $ש$ = umidade relativa; - - = radiância; --口-- = temperatura média). Barras verticais indicam o erro padrão da média.

Figure 2. Climatic data in São Luís, MA, of January of 2007 to January of 2008. ( $\boldsymbol{\square}$ = precipitation; $\mathbb{E}$ = relative humidity; $\bullet-$ = radiance; -- -- = average temperature). Vertical bars indicate the error standard of the average. 
Tabela 1. Dados quantitativos da floração, frutificação e produção de sementes em Cochlospermum orinocense.

Table 1. Quantitative data of the flowering, fruition and seed set in Cochlospermum orinocense.

\begin{tabular}{lc} 
Dados & $\begin{array}{c}\text { Média e } \\
\text { desvio padrão }\end{array}$ \\
\hline
\end{tabular}

Inflorescências/indivíduo

$10,24 \pm 3,6$

Flores/inflorescência

$247,7 \pm 100,7$

Flores abertas/inflorescência/dia

$7,2 \pm 2,3$

Flores/indivíduo

$2.536,45$

Flores abertas/indivíduo/dia

73,7

Inflorescências com frutos/indivíduo

$13,32 \pm 4,9$

Frutos/inflorescência

$17,95 \pm 4,7$

Frutos/indivíduo

Sementes/fruto

$57,91 \pm 22,8$

Sementes/indivíduo desenvolvem-se inicialmente acompanhadas de brácteas com forma semelhante às folhas, só que menores e com número menor de folíolos (figura 4A). Após a fase inicial de desenvolvimento, as brácteas caem e fica visível a inflorescência dotipo tirso(figura 4B), com o eixo principal monopodial na forma de um rácemo e as ramificações laterais na forma de cíncino, ou seja, cimeira monopodial com flores dispostas espiraladamente (figura 4C). As flores são grandes, com 85,4 \pm 2,96 mm de diâmetro, hermafroditas, amarelas e levemente zigomorfas, sendo a zigomorfia determinada pela disposição ligeiramente assimétrica das pétalas e das anteras ao redor do gineceu (figura 4D). Cada ovário possui em média $250 \pm$ 42,2 óvulos e o androceu é composto por $159 \pm 10,64$ estames com anteras poricidas. As flores apresentam em média 3.822.625,4 grãos de pólen, 81,6\% dos quais viáveis, sendo a razão $\mathrm{P} / \mathrm{O}$ de $16.019,2$.

C. orinocense produz flores com duração de um dia com a antese iniciando antes do amanhecer, entre 4h00 e 4h30, estando cerca de $80 \%$ das flores já totalmente abertas às 7h00. A receptividade dos estigmas e a abertura dos poros nas anteras acompanham a antese, com mais de 70\% das flores já receptivas e potencialmente liberando

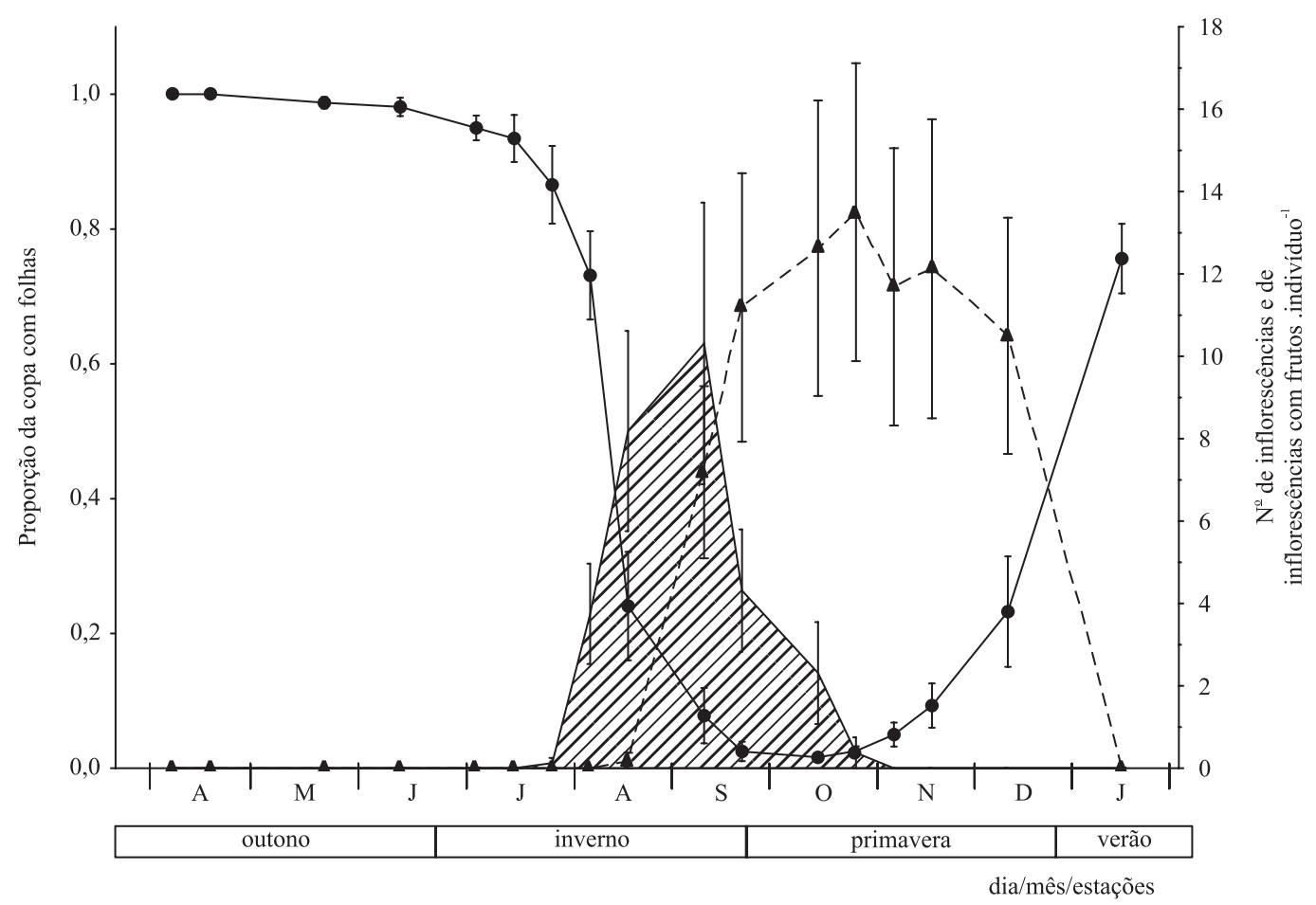

Figura 3. Dados fenométricos, de abril de 2007 a janeiro de 2008, da população de Cochlospermum orinocense na borda de mata primária. (-- = folhas; ஐ = inflorescências; -^- = inflorescências com frutos). Barras verticais indicam o erro padrão da média.

Figure 3. Fenometric data of April of 2007 to January of 2008 in Cochlospermum orinocense population in primary forest edge. ( $\bullet-=$ leaves; $\llbracket$ = inflorescences; - $\mathbf{-}-=$ inflorescences with fruits). Vertical bars indicate the error standard of the average. 

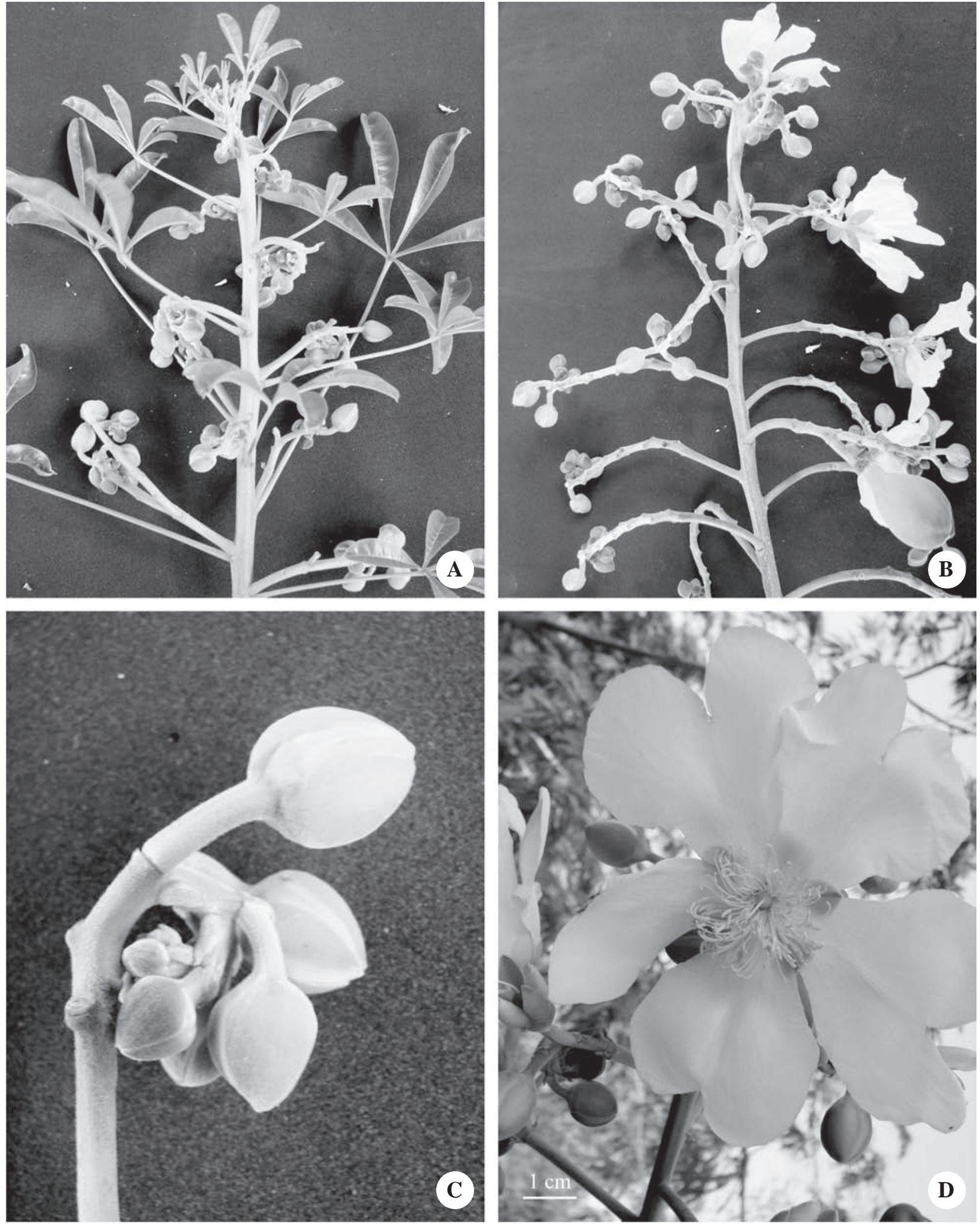

Figura 4. Cochlospermum orinocense. A. Inflorescência jovem. B. Inflorescência totalmente formada. C. Detalhe de ramo da inflorescência. D. Detalhe da flor.

Figure 4. Cochlospermum orinocense. A. Young inflorescence. B. Total formed inflorescence. C. Detail of branch of the inflorescence. D. Detail of the flower. 
pólen entre 6 h00 e 7h00, horário em que se iniciam as visitas das abelhas. A freqüência de visitas aumenta linearmente durante a manhã atingindo o máximo entre $9 \mathrm{~h} 00$ e $11 \mathrm{~h} 00$, com cerca de 40 indivíduos/hora, diminuindo então gradativamente, sendo mínima a partir das 15h00, com cerca de dois indivíduos/hora. A senescência da flor, que se manifesta pela abscisão de corola e estames, ocorreu precocemente em flores não fecundadas, entre $16 \mathrm{~h} 00$ e $19 \mathrm{~h} 00$ (senescência precoce ocorreu em $100 \%$ das flores ensacadas e em $48 \%$ das flores expostas ao polinizador). Em flores fecundadas, a abscisão iniciou-se entre 01h00 e 02h00 do dia seguinte, completando-se às $04 \mathrm{~h} 00$ em cerca de $80 \%$ das flores (figura 5).

As flores de $C$. orinocense foram visitadas por sete espécies de abelhas, distribuídas em quatro gêneros, das tribos Centridini, Meliponini e Xylocopini (tabela 2). Centris caxiensis apresentou o maior percentual de visitas (53,8\%), seguida de Centris tarsata (37,3\%). As abelhas, exceto as pertencentes ao gênero Trigona, tocavam os órgãos reprodutivos com o ventre e vibravam para retirar os grãos de pólen das anteras. Trigona fulviventris e Trigona pallens cortavam as anteras para retirar o pólen e não tocavam os estigmas das flores.
Tabela 2. Abelhas visitantes de Cochlospermum orinocense. Freqüência e resultado de visitas.

Table 2. Visiting bees of Cochlospermum orinocense. Frequency and visiting results.

\begin{tabular}{ccc}
\hline Tribo/Espécies & $\begin{array}{c}\text { Freqüência } \\
(\%)\end{array}$ & $\begin{array}{c}\text { Resultado } \\
\text { da visita }\end{array}$ \\
\hline
\end{tabular}

\section{Centridini}

$\begin{array}{lrl}\text { Centris caxiensis Ducke } & 53,8 & \text { Polinização } \\ \text { C. tarsata Smith } & 37,3 & \text { Polinização } \\ \text { C. flavifrons Fabricius } & 1,9 & \text { Polinização }\end{array}$

Meliponini

$\begin{array}{lll}\text { Melipona sp. } & 1,9 & \text { Polinização } \\ \text { Trigona fulviventris Guérin } & 2,5 & \text { Pilhagem } \\ \text { Trigona pallens Fabricius } & 1,3 & \text { Pilhagem }\end{array}$

Xylocopini

Xylocopa frontalis Olivier $\quad 1,3 \quad$ Polinização

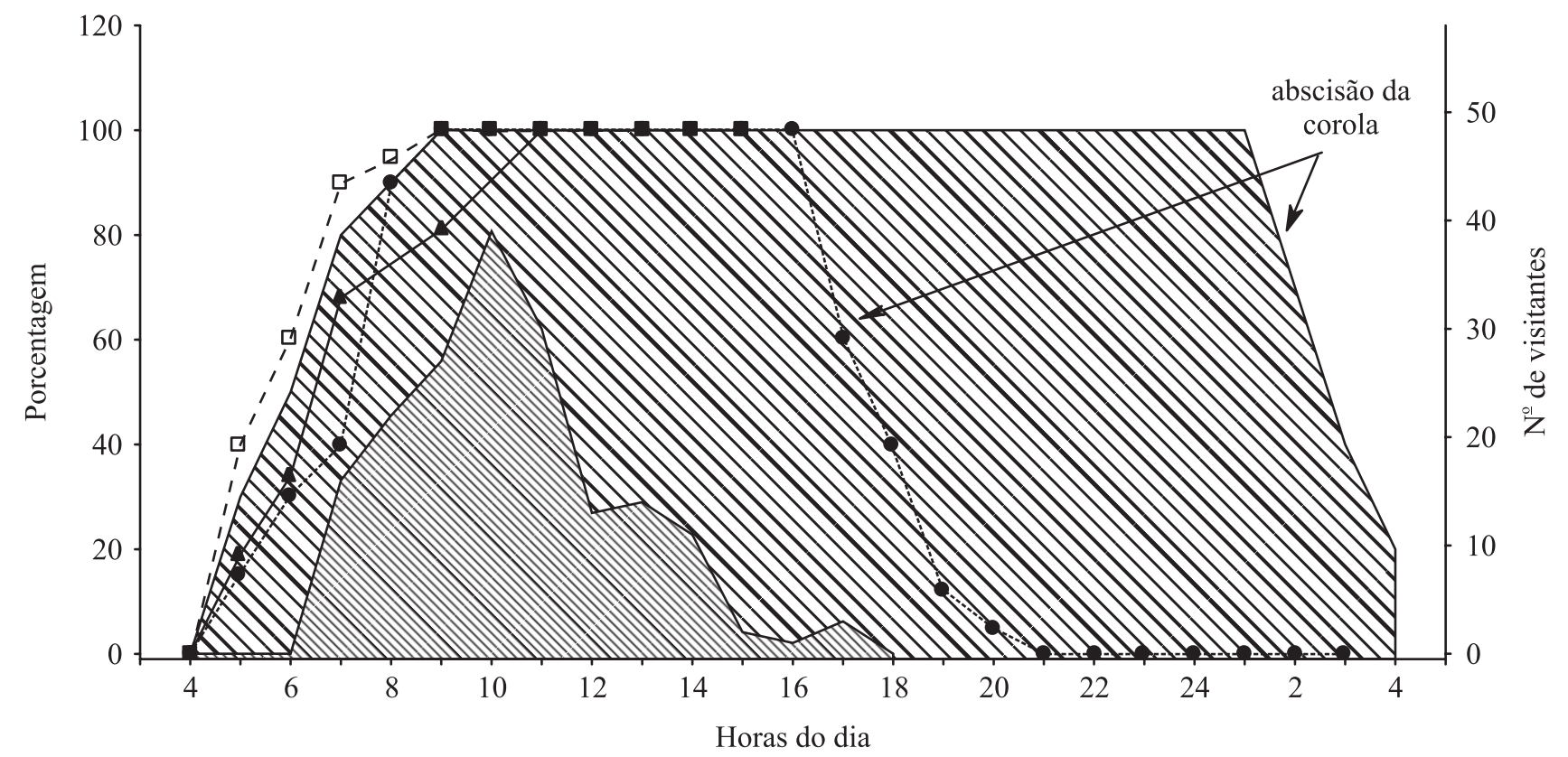

Figura 5. Longevidade das flores, receptividade do estigma, abertura dos poros nas anteras e frequência de visitantes florais em Cochlospermum orinocense. ( $₫$ = flores abertas (fecundadas); - - - = flores abertas (não fecundadas); - - - = estigmas receptivos; $\_-=$anteras poricidas abertas; $\mathbb{\mathbb { N }}$ = visitantes florais).

Figure 5. Flower longevity, stigma receptivity, opening of the pores in anthers and frequency of floral visitors in Cochlospermum orinocense. ( $₫=$ open flowers (fecundates); - - -- = open flowers (not fecundates); - - -- = receptives stigmas; $\mathbf{-}=$ open poricidals anthers; $\mathbb{\mathbb { N }}$ = floral visitors). 
Cochlospermum orinocense é autocompatível (IAI $=0,98)$ com alta eficácia reprodutiva $(\mathrm{ER}=0,81)$, exibindo taxas de sucesso reprodutivo equivalentes quando as flores polinizadas naturalmente são comparadas às polinizadas artificialmente por autofecundação, geitonogamia ou xenogamia $\left(\chi^{2}=5,84\right.$, $g l=3, N=25, P<0,12)$. Poucos frutos foram formados por autofecundação espontânea ou apomixia e estes abortaram precocemente (tabela 3).

Na tabela 3 observa-se ainda que a taxa de aborto de frutos foi alta, quando formados por geitonogamia (45\%) ou autofecundação manual (33\%), sendo praticamente nula na polinização natural e xenogamia. Na geitonogamia, a média do número de sementes produzidas por fruto $(38,62)$ foi significativamente menor quando comparada aos demais tratamentos cujos frutos produziram sementes $(H=15,24, g l=3, N=2724, P=0,0042)$. A massa das sementes formadas por autofecundação manual foi estatísticamente menor que a dos demais tratamentos $(H=17,95, g l=3, N=2724$, $P=0,0005)$.

Tabela 3. Resultados dos tratamentos de polinizações controladas em Cochlospermum orinocense. Letras diferentes indicam diferenças significativas entre tratamentos $\operatorname{com} P<0,05$.

Table 3. Results of the treatments of controlled pollinations in Cochlospermum orinocense. Different letters indicate significant differences between treatments $(P<0,05)$.

\begin{tabular}{lcccccc}
\hline Tratamento & $\begin{array}{c}\text { №. de flores } \\
\text { tratadas }\end{array}$ & $\begin{array}{c}\text { Frutos } \\
\text { iniciados }\end{array}$ & $\begin{array}{c}\text { Frutos } \\
\text { desenvolvidos }\end{array}$ & $\begin{array}{c}\text { \% de } \\
\text { sucesso }\end{array}$ & $\begin{array}{c}\text { № de } \\
\text { sementes fruto }{ }^{-1}\end{array}$ & $\begin{array}{c}\text { Massa das } \\
\text { sementes } \\
(\mathrm{mg})\end{array}$ \\
\hline $\begin{array}{l}\text { Polinização natural (controle) } \\
\text { Autopolinização espontânea }\end{array}$ & 25 & 13 & 13 & $52^{\mathrm{ab}}$ & $57,91^{\mathrm{a}} \pm 22,8$ & $34,63^{\mathrm{a}} \pm 2,7$ \\
Autopolinização manual & 28 & $2^{*}$ & 0 & $0^{\mathrm{c}}$ & - & - \\
Geitonogamia & 25 & 18 & 12 & $48^{\mathrm{ab}}$ & $59,64^{\mathrm{a}} \pm 11,10$ & $34,28^{\mathrm{b}} \pm 3,27$ \\
Xenogamia & 25 & 18 & 10 & $40^{\mathrm{b}}$ & $38,62^{\mathrm{b}} \pm 17,61$ & $34,88^{\mathrm{a}} \pm 2,76$ \\
Apomixia & 25 & 17 & 16 & $64^{\mathrm{a}}$ & $60,66^{\mathrm{a}} \pm 10,89$ & $34,64^{\mathrm{a}} \pm 3,0$ \\
\hline
\end{tabular}

* Frutos abortados muito precocemente. * Fruits aborted too soon.

\section{Discussão}

Cochlospermun orinocense é uma espécie decídua tolerante à seca (sensu Holbrook et al. 1995) com fase reprodutiva concentrada na primavera, no auge do longo período de estiagem, cuja duração é de 6 meses. A floração precoce, intensa e sincronizada na seca, atrai uma quantidade expressiva de abelhas de médio e grande porte como Centris caxiensis Ducke e C. tarsata Smith, seus principais polinizadores. Estas espécies forrageiam eficientemente entre flores do mesmo indivíduo e o fazem em vários indivíduos agrupados, padrão mais freqüente de distribuição de $C$. orinocence no habitat. Enquanto a deciduidade confere maior visibilidade às flores potencializando a atração (Janzen 1967), distribuição agrupada e floração intensa e sincronizada com grande "display floral" e oferta de pólen, em dias ensolarados e quentes, favorecem a alta atividade das abelhas. A alta razão pólen/óvulo, equivalente à de várias espécies anemófilas (Anderson et al. 2001) e a de espécies xenógamas sucessionais tardias (Cruden 1977) aumenta, como nestas, a probabilidade de polinização.

Suas flores hermafroditas estão dispostas em inflorescências do tipo tirso, conforme definições de Bell (1991) e Weberling (1992), e não panículas como descrito na literatura clássica (Eichler 1871). Elas abrem-se ao amanhecer, tornando-se imediatamente férteis, tendo longevidade de um dia. Este padrão de longevidade é o mais freqüente nas flores hermafroditas de espécies alógamas das florestas tropicais com floração sazonal intensa e sincronizada (Gentry 1974, Primack 1985). Em C. orinocense, entretanto, as flores não fecundadas abortam precocemente ao entardecer, fato que se contrapõe à longevidade fixa, comum em flores de um dia (Primack 1985), e à senescência induzida pela polinização ou pela remoção do pólen (Proctor \& Harder 1995). A senescência precoce das flores ocorre após o 
principal período de visitação dos polinizadores e pode ser um mecanismo para economia de água na seca. $\mathrm{Na}$ congenérica $C$. vitifolium Spreng., pioneira de florestas tropicais na América Central que exibe morfologia floral e estratégia reprodutiva similares, as flores mantêm um alto potencial hídrico e transpiram intensa e continuamente na seca (Chapotin et al. 2003).

Cochlospermun orinocense é uma espécie alógama autocompatível exibindo o denominado sistema de cruzamento misto (Goodwillie et al. 2005, Knigth et al. 2005), no qual espécies com flores hermafroditas se reproduzem por autofecundação e fecundação cruzada. Autofecundação espontânea prevenida pelas anteras poricidas e apomixia rara indicam que o sucesso reprodutivo depende exclusivamente da atividade do polinizador, sendo a fecundidade proporcional à disponibilidade de polinizadores.

A prevalência da limitação de pólen nas populações de plantas alógamas, causada por limitação de polinizadores e/ou insuficiência de pólen, tem sido evidenciada na maioria das espécies estudadas e baseia-se principalmente nos experimentos de suplementação, nos quais pólen extra fornecido aumenta a fecundidade das plantas (Knigth et al. 2005). A limitação aumenta com a diminuição da densidade e pode variar entre populações e temporalmente, sendo considerada a causa primária da redução na eficiência reprodutiva e fecundidade das espécies alógamas (Ashman et al. 2004, Knigth et al. 2005).

Cochlospermun orinocense exibe alta eficácia reprodutiva $(E R=0,81)$, superior à média das espécies alógamas autocompatíveis $(\mathrm{ER}=0,67)$ de florestas sazonalmente secas na Costa Rica (Bawa 1974) e à de florestas secundárias ( $\mathrm{ER}=0,66$ ) na Venezuela (Zapata \& Arroyo 1978). Sua eficácia reprodutiva é também superior à de várias espécies alógamas autocompatíveis da Floresta Atlântica e Cerrado (Proença \& Gibbs 1994, Sigrist \& Sazima 2004, Mickeliunas et al. 2006, Benezar \& Pessoni 2006). O índice (ER = polinização natural/ polinização cruzada manual) estima a fecundidade natural em função daquela obtida em condições de máxima polinização, sendo um reflexo da eficiência do polinizador (Zapata \& Arroyo 1978). A alta eficácia reprodutiva e máxima razão semente/óvulo sob polinização natural indicam que houve ampla disponibilidade de pólen e que a fecundidade na população de $C$. orinocense, no período em questão, foi limitada principalmente pelos recursos da planta mãe como prevê a teoria da seleção sexual (Bateman 1948, Janzen 1977, Willson \& Burley 1983, Wilson et al. 1994, Knigth et al. 2005). Esta limitação impõe que cerca de apenas $10 \%$ das flores produzidas originarão frutos que atingirão a maturidade (razão fruto/flor = 0,094), com a grande maioria das flores exercendo a função masculina (Sutherland \& Delph 1984) atuando como doadoras de pólen.

Fecundidade limitada principalmente pelos recursos da planta foi também observada na autoincompatível Cochlospermum vitifolium, sendo sua relativamente baixa razão semente/óvulo $(0,13)$ atribuída ao efeito do pólen incompatível (Snow \& Roubik 1987, Chapotin et al. 2003), que pode limitar a disponibilidade de cruzamentos e intensificar os efeitos da limitação de pólen (Byers \& Meagher 1992, Knight et al. 2005). A maior razão semente/óvulo em $C$. orinocense $(0,23)$ reforça a hipótese de que autocompatibilidade induz maior eficiência reprodutiva ao eliminar os efeitos deletérios do pólen incompatível.

A autocompatibilidade em $C$. orinocense é alta (IAI =0,98), com autogamia ocorrendo quando o polinizador transfere o pólen da antera ao estigma da própria flor (autofecundação facilitada) ou entre flores do mesmo indivíduo (geitonogamia). Ambas as formas de endogamia induzem a depressão endogâmica que se manifesta como maior taxa de aborto de frutos, redução do número ou da massa das sementes, reafirmando a hipótese de que a depressão endogâmica impõe limites à eficiência reprodutiva das espécies autocompatíveis, opondo-se mesmo à evolução da autocompatibilidade em plantas (Knigth et al. 2005).

Enquanto a autofecundação facilitada pode ser limitada pela separação espacial entre anteras e estigma, o comportamento do polinizador, visitando flores sucessivamente no mesmo indivíduo, sugere que geitonogamia seja a forma mais freqüente de endogamia. A prevalência de geitonogamia tem sido identificada na maioria das espécies com sistema de cruzamento misto estudadas (Schoen \& Lloyd 1992, Leclerc-Potvin \& Ritland 1994, Eckert 2000, Karron et al. 2004). Sua taxa é uma função do número de flores simultâneamente abertas ("display floral”) e da freqüência de movimento do polinizador entre flores do mesmo indivíduo (Harder \& Barrett 1995, Eckert 2000, Karron et al. 2004), ambos acentuados em $C$. orinocense.

A geitonogamia em C. orinocense induz forte depressão endogâmica provocando aborto de 45\% dos frutos e redução de $33 \%$ das sementes por fruto, aproximadamente. Não obstante, seu efeito na eficiência reprodutiva na população foi pequeno já que, sob polinização natural, a taxa de aborto de frutos foi nula e obteve-se a máxima razão semente/óvulo, embora mais 
variável (maior desvio da média no número de sementes/ fruto sob polinização natural quando comparado ao de polinizações artificiais) possivelmente devido a uma baixa freqüência de geitonogamia nas flores naturalmente polinizadas.

A geitonogamia assegura a reprodução quando cruzamentos (entre indivíduos) são escassos, mas sua taxa diminui com o aumento da densidade populacional (Murawski \& Hamrick 1991, Franceschinelli \& Bawa 2000, Ashman et al. 2004), sugerindo que a distribuição agrupada e alta densidade populacional de $C$. orinocense, favorecendo a alogamia, devam minimizar os efeitos da depressão endogâmica. De acordo com Eckert (2000) e Ashman et al. (2004), a manutenção de alta taxa de alogamia com endogamia inversamente proporcional à densidade das plantas combina heterose, em indivíduos agrupados, com maior segurança na produção de sementes por indivíduos isolados.

A alta fecundidade em C. orinocense resulta, portanto, da sua eficiente estratégia reprodutiva que combina distribuição agrupada e floração intensa e sincronizada na seca, em árvores decíduas, com alta razão pólen/óvulo, eficiente polinização por abelhas de médio ou grande porte e sistema de cruzamento misto. Esta estratégia permite que fecundidade seja limitada pelos recursos da planta e não por pólen; reduz a geitonogamia, minimizando a depressão endogâmica em populações com distribuição agrupada e pode assegurar a reprodução em indivíduos isolados na floresta.

Agradecimentos - Ao biólogo, pedagogo e amigo José Maria dos Reis Maia Filho, cuja criatividade e participação foram decisivas para a captura das abelhas. À Merck S.A., filial São Luís, pelo apoio logístico.

\section{Referências bibliográficas}

ANDERSON, G.J, BERNARDELLO, G., STUESSY, T.F. \& CRAWFORD, D.J. 2001. Breeding system and pollination of selected plants endemic to Juan Fernández islands. American Journal of Botany 88:220-233.

ASHMAN, T., KNIGHT, T.M., STEETS, J.A., AMARASEKARE, P., BURD, M., CAMPBELL, D.R., DUDASH, M.R., JOHNSTON, M.O., MAZER, S.J., MITCHELL, R.J., MORGAN, M.T. \& WILSON, W.G. 2004. Pollen limitation of plant reproduction: ecological and evolutionary causes and consequences. Ecology 85:2408-2421.

BATEMAN, A.J. 1948. Intra-sexual selection in Drosophila. Heredity 2:349-68.

BAWA, K.S. 1974. Breeding systems of tree species of a lowland tropical community. Evolution 28:85-92.
BAWA, K.S. 1992. Mating systems, genetic differentiation and speciation in tropical forest plants. Biotropica 24:251-255.

BELL, A.D. 1991. Plant form: an illustrated guide to flowering plant morphology. Oxford Universiy Press. Oxford.

BENEZAR, R.M.C. \& PESSONI, L.A. 2006. Biologia floral e sistema reprodutivo de Byrsonima coccolobifolia (Kunth) em uma savana amazônica. Acta Amazonica 36:159-168.

BOND, W.J. 1994. Do mutualisms matter? Assessing the impact of pollinator and disperser disruption on plant extinction. Philosophical Transactions of the Royal Society of London 344:83-90.

BYERS, D.L. \& MEAGHER, T.R. 1992. Mate availability in small populations of plant species with homomorphic sporophytic self incompatibility. Heredity 68:353-59.

CHAPOTIN, S.M., HOLBROOK, M.N., MORSE, S.R. \& GUTIERREZ, M.V. 2003. Water relations for tropical dry forest flowers: patways for water entry and the role of extracellular polyssaccarides. Plant Cell and Environment 26:623-630.

CHAZDON, R.L., CAREAGA, S., WEBB, C. \& VARGAS, O. 2003. Community and phylogenetic structure of reproductive traits of woody species in wet tropical forests. Ecological Monographs 73:331-348.

CRUDEN, R.W. 1977. Pollen-ovule ratios: a conservative indicator of breeding systems in flowering plants. Evolution 31:32-46.

ECKERT, C.G. 2000. Contributions of autogamy and geitonogamy to self-fertilization in a mass flowering, clonal plant. Ecology 81:532-542.

EICHLER, A.W. 1871. Bixaceae. In Flora Brasiliensis. (C.F.P. Martius \& A.G. Eichler, eds.). F.Fleischer, Lipsiae, v.13, pars 55, p.421-526.

FOURNIER, L.A. 1974. Un método cuantitativo para la medición de características fenológicas en árboles. Turrialba 24:422-423.

FRANCESCHINELLI, E.V. \& BAWA, K.S. 2000. The effect of ecological factors on the mating system of a South American shrub species (Helicteres brevispira). Heredity 84:116-123.

FRANKIE, G.W. \& COVILLE, R.E. 1979. An experimental study on the foraging behavior of selected solitary bee species in the Costa Rican dry forest. Journal of the Kansas Entomological Society 52:591-602.

FRANKIE, G.W., OPLER, P.A. \& BAWA, K.S. 1976. Foraging behavior of solitary bees:implications for outcrossing of a neotropical forest tree species. Journal of Ecology 64:1049-1057.

FRANKIE, G.W., VINSON, S.B., NEWSTROM, L.E. \& BARTHELL, J.F. 1988. Nest site and habitat preferences of Centris bees in the Costa Rica dry forest. Biotropica 20:301-310.

GENTRY, A.H. 1974. Flowering phenology and diversity in tropical Bignoniaceae. Biotropica 6:64-69. 
GOODWILLIE, C., KALISZ, S. \& ECKERT, C.G. 2005. The evolutionary enigma of mixed mating systems in plants: occurrence, theoretical explanations and empirical evidence. Annual Review of Ecology Evolution and Systematics 36:47-79.

HARDER, L.D. \& BARRETT, S.C.H. 1995. Mating cost of large floral displays in hermaphrodite plants. Nature 373:512-515.

HOLBROOK, N.M., WHITBECK. J.L. \& MOONEY, H.A. 1995. Drought responses of neotropical dry forest trees. In Seazonally dry tropical forest (S.H. Bullock, H.A Mooney \& E. Medina, eds.). Cambridge University Press, New York, p.243-270.

JAMES, T., VEGE, S., ALDRICH, P. \& HAMRICK, J.L. 1998. Mating systems of three tropical dry forest tree species. Biotropica 30:587-594.

JANZEN, D.H. 1967. Synchronization of sexual reproduction of trees within the dry season in Central America. Evolution 21:620-637.

JANZEN, D.H. 1977. A note on optimal mate selection in plants. American Naturalist 111:365-71.

KARRON, J.D., MITCHELL, R.J., HOLMQUIST, K.G., BELL, J.M. \& FUNK, B. 2004. The influence of floral display size on selfing ratesb in Mimulus ringens. Heredity 92:242-248.

KEATING, R.C. 1972. The comparative morphology of the Cochlospermaceae. III. The flower and pollen. Annals of the Missouri Botanical Garden 59:282296.

KNIGHT, T.M., STEETS, J.A., VAMOSI, J.C., MAZER, S.J., BURD, M., CAMPBELL, D.R., DUDASH, M.R., JOHNSTON, M.O., MITCHELL, R.J. \& ASHMAN, T. 2005. Pollen limitation of plant reproduction: pattern and process. Annual Review of Ecology Evolution and Systematics 36:467497.

KOELEWIJN, H.P. 1998. Effects of different levels of inbreeding on progeny fitness in Plantago coronopus. Evolution 52:692-702.

LECLERC-POTVIN, C. \& RITLAND, K. 1994. Modes of self- fertilization in Mimulus guttatus (Scrophulariaceae): a field experiment. American Journal of Botany 81:199205.

LLOYD, D.G. 1972. Breeding systems in Cotula L. (Compositae, Anthemidae). New Phytologist 71:11811194.

MACFARLANE, T.D. 2007. Florabase - The Western Australian Flora. Western Australian Herbarium, Department of Environment and Conservation. http:// florabase.calm.wa.gov.au/browse/profile/21792. (acesso em 25/05/2008).

MAÊDA, J.M. 1985. Manual para uso da câmara Neubauer para contagem de pólen em espécies florestais. Universidade Federal do Rio de Janeiro, Rio de Janeiro.
MICKELIUNAS, L., PANSARIN, E.R. \& SAZIMA, M. 2006. Biologia floral, melitofilia e influência de besouros Curculionidae no sucesso reprodutivo de Grobya amherstiae Lindl. (Orchidaceae: Cyrtopodiinae). Revista Brasileira de Botânica 29:251-258.

MURAWSKI, D.A. \& HAMRICK, J.L. 1991. The effect of the density of flowering individuals on the mating systems of 9 tropical tree species. Heredity 67:167-174.

NORONHA, M.R.P. \& GOTTSBERGER, G. 1980. A polinização de Aspilia floribunda (Asteraceae) e Cochlospermum regium (Cochlospermaceae) e a relação das abelhas visitantes com outras plantas do Cerrado de Botucatu, Estado de São Paulo. Revista Brasileira de Botânica 3:67-77.

OPLER, P.A., BAKER, H.G. \& FRANKIE, G.W. 1980. Plant reproductive characteristics during secondary succession in neotropical lowland forest ecosystems. Biotropica 12:40-46.

PENNINGTON, R.T., PRADO, D.E. \& PENDRY, C.A. 2000. Neotropical seasonally dry forest and Quaternary vegetation changes. Journal of Biogeography 27:261273.

PRIMACK, R.B. 1985. Longevity of individual flowers. Annual Review of Ecology and Systematics 16:15-37.

PROCTOR, H.C. \& HARDER, L.D. 1995. Effect of pollination success on floral longevity in the orchid Calypso bulbosa (Orchidaceae). American Journal of Botany 82:1131-1136.

PROENÇA, C.E.B. \& GIBBS, P.E. 1994. Reproductive biology of eight sympatric Myrtaceae from Central Brazil. New Phytologist 126:343-354.

RADFORD, A.E, DECKINSON, W.C., MASSEY, J.R. \& BELL, C.R. 1974. Vascular plant systematics. Harper \& Row Publishers, New York.

ROBINSOHN, I. 1924. Die Farbungsreaktion der Narbe, Stigmatochromie, als Morpho-biologische Blütenuntersuchungsmethode. Sitzungsberichten Akademischer Wissenschaft Wien, Mathematics, Abteilung 133:180-213.

SCHOEN, D.J. \& LLOYD, D.G. 1992. Self and crossfertilization in plants. III. Methods for studying modes and functional aspects of self-fertilization. International Journal of Plant Sciences 153:381-393.

SIGRIST, M.R.A. \& SAZIMA, M. 2004. Pollination and reproductive biology of twelve species of Neotropical Malpighiaceae: stigma morphology and its implications for the breeding system. Annals of Botany 94:33-41.

SNOW, A.A. \& ROUBIK, D.W. 1987. Pollen deposition and removal by bees visiting two tree species in Panama. Biotropica 19:57-63.

SUTHERLAND, S. \& DELPH, L.F. 1984. On the importance of male fitness in plants: patterns of fruit-set. Ecology 65:1093-1104. 
USDA. 2006. National Genetic Resources Program. Germplasm Resources Information Network - (GRIN) [Online Database]. National Germplasm Resources Laboratory, Beltsville, Maryland. URL. http://www. ars-grin.gov/cgi-bin/npgs/html/taxon.pl 11018 (acesso em 25/05/2008).

WEBERLING, F. 1992. Morphology of flowers and inflorescences. Cambridge University Press. Cambridge.

WILLSON, M.F. \& BURLEY, N. 1983. Mate choice in plants. Princeton University Press. Princeton.
WILSON, P., THOMPSON, J.D. \& STANTON, M.L. 1994. Beyond floral Batemania: gender biases in selection for pollination success. American Naturalist 143:283-96.

WOODSON, R.E., SCHERY, R.W. \& ROBYNS, A. 1967. Flora of Panama. Part VI. Family 126. Cochlospermaceae. Annals of the Missouri Botanical Garden 54:61-64.

ZAPATA, T.R. \& ARROYO, M.T.K. 1978. Plant reproductive ecology of a secondary deciduous tropical forest in Venezuela. Biotropica 10:221-230.

ZAR, J.H. 1996. Biostatistical analyses. Prentice-Hall, New Jersey. 